\title{
SVM-Based Face Recognition Using Genetic Search for Frequency-Feature Subset Selection
}

\author{
Aouatif Amine, Mohammed Rziza, and Driss Aboutajdine \\ GSCM-LRIT, Faculty of Sciences, Mohammed V University, \\ B.P. 1014 Rabat, Morocco \\ aouatif.amine@ieee.org, \{rziza, aboutaj\}@fsr.ac.ma
}

\begin{abstract}
The goal of this paper is to study if there is a dependency between a selected feature vector at each generation of the genetic algorithm and the resulting fitness. In order to see the relation between these parameters, we first use Discrete Cosine Transforms (DCT) to transform each image as a feature vector (i.e., Frequency Feature Subset (FFS)). A Genetic Algorithm (GA) is then used to select a subset of features from the low-dimensional representation by removing certain DCT coefficients that do not seem to encode important information about recognition task. When using SVM, two problems are confronted: how to choose the optimal input feature subset for SVM, and how to set the best kernel parameters. Therefore, obtaining the optimal feature subset and SVM parameters must occur simultaneously. We present a genetic algorithm approach for feature selection and parameters optimization to solve this kind of problem.
\end{abstract}

mots clef- Face recognition, Feature Selection, Genetic Algorithm, Support Vector Machine, Discrete Cosine Transform.

\section{Introduction}

Machine recognition of faces is becoming more and more popular and the need for accurate and robust performance is increasing. Face recognition, as an unsolved problem under the conditions of pose and illumination variations, still attracts significant research efforts. The main reasons for the ongoing research are: (i) the increased need for natural identification for authentication in the networked society, for surveillance, for perceptual user interfaces, (ii) and the lack of robust features and classification schemes for the face recognition task.

A common objective in face recognition is to find a good way of representing face information. High information redundancy present in face images results in inefficiencies when these images are used directly for recognition, identification and classification. A key point in developing a good representation is to expose the constraints and remove the redundancies contained in pixel images of faces. Typically one builds a computational model to transform pixel images into face features, which generally should be robust to variations of illumination, scale and orientation and then use these features for recognition [1]. For classical pattern recognition techniques, the patterns are generally represented as a vector of feature values. The selection of features can have a considerable impact on the effectiveness of the resulting classification algorithm [5]. It is

A. Elmoataz et al. (Eds.): ICISP 2008, LNCS 5099, pp. 321-328. 2008.

(C) Springer-Verlag Berlin Heidelberg 2008 
not often known in advance wich features will provide the best discrimination between classes, and it is usually not feasible to measure and represent all possible features of the objects effects. With feature selection, the cost of classification can be reduced by limiting the number of features which must be measured and stored. Some, but not all, feature selection methods realize this benefit as well. A number of approaches for feature subset selection have been proposed in the literature. Koller et al [2] used a greedy algorithm to remove the features that provide the least additional information given the remaining features. Brill et. al [3] have explored randomized population-based heuristic search approaches such as GAs to select feature subsets for NNs. As is known, in many supervised learning problems, feature selection is important, and for SVM, it also performs badly when there are many irrelevant features [4]. In order to improve its performance, suitable feature selection algorithm, such as MLR (Multiple Linear Regression), GA, should be adopted. GAs are good candidates for attacking this challenge since GAs are very useful for extracting patterns in multiclass, high-dimensionality problems where heuristic knowledge is sparse or incomplete. The kernel parameters setting for SVM in a training process impacts on the classication accuracy. Feature selection is another factor that impacts classication accuracy. In this paper, the basic idea here consists in using a GA to optimize the parameters and to discover good subsets of genes simultaneously, without degrading the SVM classication accuracy. The GA feature extractor presented here utilizes feedback from the SVM classifier to the feature extractor Fig. 1 in section 5 .

\section{Discrete Cosine Transform}

High information redundancy and correlation in face images result in inefficiencies when such images are used directly for recognition. DCT is a predominant tool first introduced by Ahmed et al. [7]. Since then, it was widely used as a feature extraction and compression in various applications on signal and image processing and analysis due to its fine properties, i.e., de-correlation, energy compaction, separability, symmetry and orthogonality. In face recognition, DCTs are used to reduce image information redundancy because only a subset of the transform coefficients are necessary to preserve the most important facial features.

\section{SVM Classifier for Face Recognition}

Recently, the SVM has been gaining popularity in the field of pattern classification due to its promising empirical performance, moderate computation complexity and its strong mathematical foundation. SVM are binary classifiers and different approaches like "one-against-all" and "one-against-one" are built to extend SVM to the multi-class classification case for face recognition [10]. The major method is the "one-against-one" method. This method constructs classifiers where each one is trained on data from two classes. For training data from the $i^{t h}$ and the $j^{t h}$ classes, we solve the following binary classification problem:

$$
\min _{w^{i j}, b^{i j}, \xi^{i j}} \frac{1}{2}\left(w^{i j}\right)^{T} w^{i j}+C \sum_{t} \xi_{t}^{i j}\left(w^{i j}\right)^{T}
$$




$$
\begin{array}{r}
\left(w^{i j}\right)^{T} \phi\left(x_{t}\right)+b^{i j} 1-\xi_{t}^{i j}, \text { if } y_{t}=i \\
\left(w^{i j}\right)^{T} \phi\left(x_{t}\right)+b^{i j} 1-\xi_{t}^{i j}, \text { if } y_{t}=i \\
\xi_{t}^{i j} \geq 0
\end{array}
$$

There are different methods for doing the future testing after all $p(p-1) / 2$ classifiers are constructed. After some tests, we decide to use the following voting strategy suggested in [13]: if $\operatorname{sign}\left(\left(w^{i j}\right)^{T} \phi\left(x_{t}\right)+b^{i j}\right)$ says $x$ is in the $i^{\text {th }}$ class, then the vote for the $i^{\text {th }}$ class is added by one. Otherwise, the $j^{\text {th }}$ is increased by one. Then we predict $x$ is in the class with the largest vote. The voting approach described above is also called the Max Wins strategy. In case that two classes have identical votes, thought it may not be a good strategy, now we simply select the one with the smaller index. Practically we solve the dual of (Eq.11) whose number of variables is the same as the number of data in two classes. Hence if in average each class has $l / k$ data points, we have to solve $k(k-1) / 2$ quadratic programming problems where each of them has about $2 l / k$ variables.

\section{Genetic Algorithm for SVM Parameters Optimization}

Goldberg [15] provides a nice introduction to GAs and the reader is referred to this source as well as the survey paper of Srinivas and Patnaik [8] for further information. The genetic algorithm is a method for solving both constrained and unconstrained optimization problems that is based on natural selection, the process that drives biological evolution. The genetic algorithm repeatedly modifies a population of individual solutions. At each step, the genetic algorithm selects individuals at random from the current population to be parents and uses them to produce the children for the next generation. Over successive generations, the population "evolves" toward an optimal solution. The genetic algorithm uses three main types of rules at each step to create the next generation from the current population: Selection, Crossover, and Mutation. In the literature, only a few algorithms have been proposed for SVM feature selection like in [9]. Some other GA-based feature selection methods were proposed [11,12]. However, these papers focused on feature selection and did not deal with parameters optimization for the SVM classier. Therefore, in addition to the feature selection, proper parameters setting can improve the SVM classication accuracy. The choice of $\mathrm{C}$ and the kernel parameter is important to get a good classication rate. In the most case these parameters are tuned manually. In order to automatize this choice we use genetic algorithms. The SVM parameters, $C$ and $\gamma$ are real, we have to encode them with binary chains; we fix two search intervals, one for each parameter, $C_{\max } \leq C \leq C_{\min }$ and $\gamma_{\max } \leq \gamma \leq \gamma_{\min }$. To encode $C$ and $\gamma$, we discretize the search spaces. Thus, a 32 bits encoding scheme of $C$ is given by $C_{b 1}, \ldots C_{b 32}$ where:

$$
C_{b}=\sum_{i=1}^{32} C_{b i} 2^{i-1}
$$

and $\gamma$ by $\gamma_{b 1}, \ldots \gamma_{b 32}$ where:

$$
\gamma_{b}=\sum_{i=1}^{32} \gamma_{b i} 2^{i-1}
$$


with $C_{b}=g_{\max }\left(C-C_{\min }\right) /\left(C_{\max }-C_{\min }\right)$ and $\gamma_{b}=g_{\max }\left(\gamma-\gamma_{\min }\right) /\left(\gamma_{\max }-\gamma_{\min }\right)$ and $g_{\max }=2^{32}-1$.

The fitness function used to evolve the chromosomes population is the SVM classification rate. The goal was to see if the GA would discover the work effectively. We lists some reasons why SVM must be used combined feature selection. One major weakness of SVMs is their high computational cost, which precludes real-time applications. In addition, SVMs are formulated as a quadratic programming problem and, therefore, it is difficult to use SVMs to do feature selection directly. Some researchers have proposed approximations to SVM for feature selection by first training the SVM using the whole training set, and then computing approximations to reduce the number of features.

\section{Overview of the Proposed Method}

The main steps of the proposed method are as follows:

1. FFS extraction using DCT.

2. Using Genetic Algorithms, in order to generate both the optimal feature subset and SVM parameters at the same time.

3. Classification of novel images.

Fig. 1 presents the general schema of feature selection and classification process. Firstly, a population of possible frequency features subset is genetically evolved, these features seems to be most useful to a particular classification problem from all those

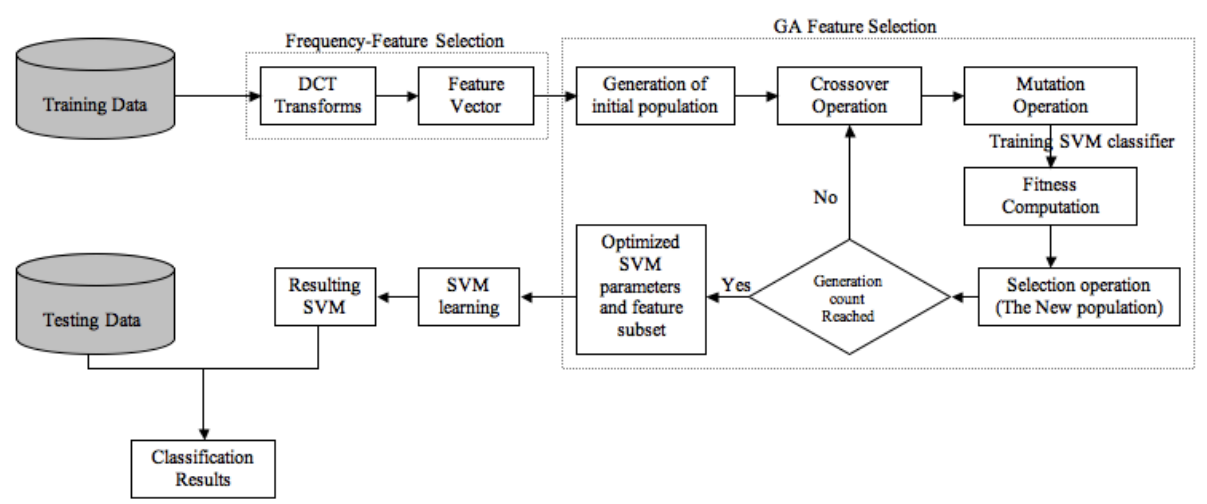

Fig. 1. The general process for gene subset selection and classification

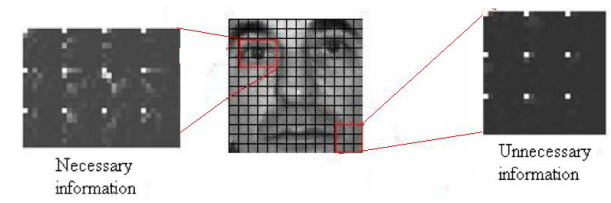

Fig. 2. Illustration of the effects of the blockbased DCT for local appearance based face representation 
available, it can be explain that they contain only highly informative and non-redundant features, which significantly improve classification. The genetic evolution is guided using the proposed fitness criterion, the quality of a given chromosome is proportional to the information gain measure computed using the dataset records retrieved from the training dataset, the chromosome comprises three parts, $\mathrm{C}$, kernel parameter, and the features mask. The result is finally validated using a new test dataset. In fact, the basic idea here consists in using a GA to discover good subsets of genes, the goodness of a subset being evaluated by SVM classifier.

Using these methods we obtained three benefits, the first one that computational complexity is reduced as there is smaller number of inputs. Often, a secondary benefit found is that the accuracy of the classifier increases, and the last one is to remove the extra features (i.e like noise, obscuring other features from the learning algorithm) from a feature set, like unnecessary information showed in Fig. 2

\section{The Dataset}

To assess the robustness of our method against different facial expressions, lighting conditions and pose, we have collect grey-scale face images from two different face database available in the public domain, ORL face database 1 and Yale face database 2 .

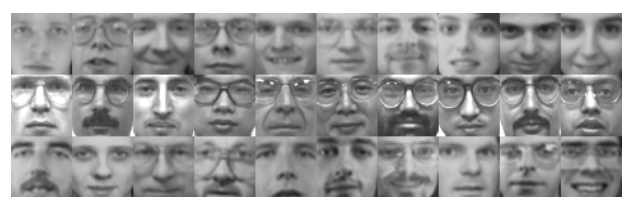

Fig. 3. Some samples from the used face database (ORL+Yale)

Face images selected are near frontal and contain variations in pose, illumination and expression. Eyebrows, eyes, nose, lips and surrounding area of face image contribute maximum in face recognition. So scale normalization of face images of data sets is carried out using the cropping phenomena which eliminate the unnecessary information from image and retain only internal structures. All the faces are then scaled to the size $48 \times 48$ pixels, aligned according to the eye positions. Sample images from the face databases are shown in Fig. 3 There are 330 subjects with 10 images per subject for a total of 3, 300 images. The entire face database (ORL + Yale) is divided into two parts, six images of each subject are used to construct the training data and the remaining images are used for testing.

\section{Experiment Results}

We have performed a number of experiments in order to demonstrate the performance of the proposed approach on gray-scale images. In our study, the local information of

\footnotetext{
${ }^{1}$ http://www.cl.cam.ac.uk/Research/DTG/attarchive:pub/data/att_faces.zip

${ }^{2} \mathrm{http} / / / \mathrm{cvc}$. yale.edu/projects/yalefaces/yalefaces.html
} 


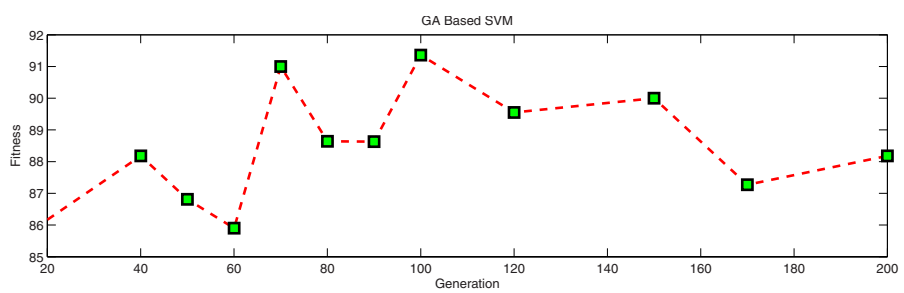

Fig. 4. The evolution of the best overall accuracy values for proposed approach

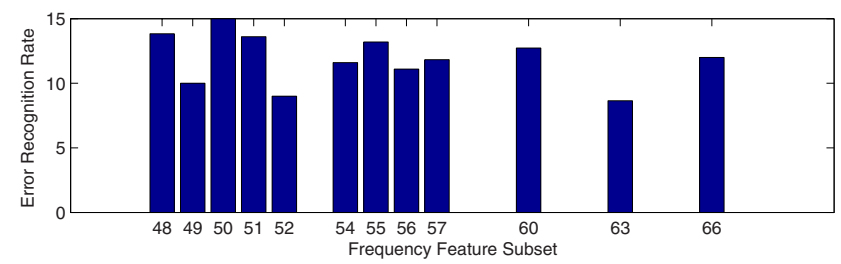

Fig. 5. Error Recognition Rate of SVM classifier as a function of the number of FFS selected by the GA approach

a candidate face can be obtained by using block-based DCT as follows: a face image is divided into blocks of 8 by 8 pixels size without overlapping. Each block is then represented by its DCT coefficients. From the obtained DCT coefficients only a small, generic feature set is retained in each block. Ekenel et al. [14] has proved that the highest information necessary to achieve high classification accuracy is contained in the first low frequency DCT coefficients via zigzag scanning.

The selection of features can have a considerable impact on the effectiveness of the resulting classification algorithm. Consider a feature set, $F=\left\{f_{0}, f_{1}, \ldots ., f_{N}\right\}$ which resulting from block-based DCT. The problem of feature selection can be seen as a case of feature weighting, where the numerical weights for each of the features have been replaced by binary values. A value of 1 could mean the inclusion of the corresponding feature into the subset, while a value of 0 could mean its absence. In a domain where objects are described by $n$ features, there are $2^{n}$ possible feature subsets. Obviously, searching exhaustively for the best subset is futile. For this reason, the genetic algorithms has been identified as the best tools to explore such search space, and produce pseudo-optimal solutions that are sufficient to produce acceptable results. We run several experiments using the parameters for the designed GA as follows: population size: 50 , crossover rate: 0.8 , mutation rate: 0.01 , and number of generations variate between 20 and 200.

As explained above, we used the GA approach to select a set of good FFS for SVMs classifier, the polynomial kernel has been found in our simulations to outperform linear and RBF kernel functions. In the present work, the library LIBSVM 3 was used with a 5 -fold cross-validation on the training data. We have computed histogram (see Figs. 5) showing the Error Recognition Rate as function of selected FFS.

\footnotetext{
${ }^{3} \mathrm{http} / / / w w w . c s i e . n t u . e d u . t w /$ cjlin/libsvm
} 
In the AG, pairs of $(\mathrm{C}, \mathrm{d})$ are tried and the one with the best cross-validation accuracy is chosen. In the test set the highest accuracy was achieved with the SVM parameters pair $(1,1.5)(\mathrm{C}$, degree of polynomial kernel), which was obtained after several trials, while varying the dimensionality of the generation. The results are presented in Fig. 4 The performance improves immensely as the number of dimensions is increased from 60 to 70 . Increasing the dimensionality from 70 to 100 provides only a relatively small improvement, while signicantly increasing the amount of computation time required to generate the models. Based on this we have chosen 70 as the dimensionality of generation. The accuracy obtained by our algorithm during feature selection was $91 \%$, using only $2.7 \%$ of the complete set of DCT coefficients under 70 generation. We can see in Fig. 4 that only 70 generation can be used to select FFS which contain a significant amount of person dependent information and got . Thus increasing the generation size to 200 implies a reduction in performance. This is verified in Fig. 5, where the vector length of FFS equal to 66 (generation size : 200) have worse performance than vector length of FFS equal to 52 (generation size : 70). We find that the 52 FFS has the maximal contribution value which is a criterion of feature importance to influence the Fitness. The pure DCT-SVM alone had an $87 \%$ recognition rate for the complete set of DCT coefficients.

\section{Conclusions and Future Works}

In conclusion, frequency feature subset based GA and SVM can further improve performance. An GA-SVM is proved to be effective in selecting FFS and significant fitness even if the sample set is very small. Thus, we have shown that genetic algorithms can play an important role in the automated loop of feature extraction and of classication. The obtained result shows that the genetic search can find very acceptable solutions for this problem in an acceptable run time, thereby obtaining a higher classification accuracy compared to DCT-SVM used only. In term of future works, this approach should be tested with other various datasets with different dimensions.

\section{Acknowledgments}

We gratefully acknowledge the support of the Maroc Telecom IAM (grant No. 105 909 05/PI) project 'Access Control' for this research. In addition, we thank Dr. Hicham LAANAYA for his contribution to the development of the GA feature extraction technique.

\section{References}

1. Chellappa, R., Wilson, C.L., Sirohey, S.: Human and machine recognition of faces: A survey. Proceedings of the IEEE 83(5), 705-740 (1995)

2. Koller, D., Sahami, M.: Towards optimal feature selection. In: ICML 1996, Bari, Italy, pp. 87-95 (1996)

3. Brill, F., Brown, D., Martin, W.: Fast genetic selection of features for neural network classifers. IEEE Transaction on Neural Networks 3(2), 324-328 (1992) 
4. Weston, J., Mukherjee, S., Chapelle, O., Pontil, M., Poggio, T., Vapnik, V.: Feature selection for support vector machines. Advances in Neural Information Processing Systems (2000)

5. Jain, A.K., Zongker, D.: Feature selection: Evaluation, application, and small sample performance. IEEE Transaction on Pattern Analysis and Machine Intelligence 19(2), 153-158 (1997)

6. Devijverand, P.A., Kittler, J.: PatternRecognition:AStatisticalApproach. Prentice Hall, Lodon (1982)

7. Ahmed, N., Natarajan, T., Rao, K.R.: Discrete Cosine Transform. IEEE Transactions Computers 23, 90-94 (1974)

8. Srinivas, M., Patnaik, L.: Genetic algorithms: A survey. IEEE Computer 27, 1726 (1994)

9. Bradley, P.S., Mangasarian, O.L.: Feature selection via concave minimization and support vector machines. In: Proceedings of the 13th international conference on machine learning, San Francisco, CA, pp. 8290 (1998)

10. Burges, J.C.: A Tutorial on Support Vector Machines for Pattern Recognition. Data Mining and Knowledge Discovery 2(2), 121-167 (1998)

11. Raymer, M.L., Punch, W.F., Goodman, E.D., Kuhn, L.A., Jain, A.K.: Dimensionality reduction using genetic algorithms. IEEE Transactions on Evolutionary Computation 4(2), 16417 (2000)

12. Yang, J., Honavar, V.: Feature subset selection using a genetic algorithm. IEEE Intelligent Systems 13(2), 4449 (1998)

13. Friedman, J.: Another approach to polychotomous classification Technical report, Department of Statistics, Stanford University (1996)

14. Ekenel, H.K., Stiefelhagen, R.: Local Appearance based Face Recognition Using Discrete Cosine Transform. In: EUSIPCO 2005, Antalya, Turkey, vol. 23(7) (2005)

15. Goldberg, D.: Genetic Algorithms in Search,Optimization,andMachine Learning. AddisonWesley, Reading (1989) 